\title{
Teste de condutividade elétrica para diferenciação dos níveis de deterioração de sementes de forrageiras
}

\author{
Adriana Hernandes Pinto ${ }^{1}$, Flávio Ferreira da Silva Binotti ${ }^{1}$, Hugo Manoel Souza ${ }^{2}$, Thiago $^{2}$ \\ Barbosa Batista ${ }^{3}$, Geraldo Cândido Cabral Gouveia ${ }^{1}$ \\ ${ }^{1}$ Universidade Estadual de Mato Grosso do Sul, Unidade Universitária de Cassilândia, Cassilândia, Mato Grosso do Sul, Brasil. E- \\ mail: adriana-hpinto@hotmail.com, binotti@uems.br, geraldocgouveia@hotmail.com \\ ${ }^{2}$ Universidade Federal de Mato Grosso do Sul, Campus Chapadão do Sul, Chapadão do Sul, Mato Grosso do Sul, Brasil. E-mail: \\ hugo_manoel12@hotmail.com \\ ${ }^{3}$ Universidade Estadual Paulista Júlio de Mesquita Filho, UNESP, Botucatu, São Paulo, Brasil. E-mail: \\ thiago_batista31@hotmail.com
}

Recebido: 15/03/2016; Aceito: 12/05/2016.

\begin{abstract}
RESUMO
O teste de condutividade elétrica destaca-se por gerar respostas rápidas para diferenciação de lotes em diferentes níveis de deterioração. No entanto, necessita-se de novos estudos visando o aprimoramento desta prática para espécies forrageiras, como Brachiaria brizantha e Panicum maximum. O objetivo foi adequar o teste de condutividade elétrica para determinação do nível de deterioração de sementes de $B$. brizantha cv. MG-5 e de $P$. maximum cv. Tanzânia. O delineamento experimental foi inteiramente casualizado e os tratamentos dispostos em esquema fatorial $3 \times 3$, sendo três níveis de deterioração e três períodos de embebição $(8,16$ e 24 horas $)$ à $25^{\circ} \mathrm{C}$, com 6 repetições. Avaliou-se a condutividade elétrica em três volumes de água da solução de embebição $(25,50$ e $75 \mathrm{~mL}$ ) e três quantidades de sementes (25, 50 e 75 sementes). Recomenda-se para Brachiaria brizantha cv. MG-5 a utilização de 25 sementes durante o período de 8 horas nos testes de condutividade elétrica, independentemente da quantidade de solução de embebição. Para Panicum maximum cv. Tanzânia é adequada a utilização de 50 sementes em $25 \mathrm{~mL}$ de solução de embebição, independente do período de embebição.
\end{abstract}

Palavras-chave: período de embebição, Brachiaria brizantha, Panicum maximum, vigor, qualidade fisiológica.

\section{Electrical conductivity test to differentiation of deteriorating levels of forage seeds}

\begin{abstract}
The electrical conductivity test stands out for generating rapid responses to differentiation of seed lots at different deterioration levels. However, there is need for further studies to improve this practice for forage species such as Brachiaria brizantha and Panicum maximum. The objective was to adjust the electrical conductivity test to differentiation of deterioration level of B. brizantha seeds MG-5 and P. maximum cv. Tanzania. The experimental design was completely randomized and the treatments arranged in a factorial $3 \times 3$, three levels of deterioration and three soaking periods $\left(8,16\right.$ and 24 hours) at $25{ }^{\circ} \mathrm{C}$ with 6 repetitions. The electrical conductivity in three water volumes of imbibition solution $(25,50$ and $75 \mathrm{~mL})$ and three amounts of seeds $(25,50$, and 75 seeds) were measured. It is recommended to Brachiaria brizantha cv. MG-5 using 25 seeds within 8 hours on electrical conductivity, regardless of the amount of soaking solution. For Panicum maximum cv. Tanzania using 50 seeds in $25 \mathrm{ml}$ of soaking solution independent of the soaking period is appropriate.
\end{abstract}

Key words: soaking period, Brachiaria brizantha, Panicum maximum, force, physiological quality 


\section{Introdução}

Diversas são as espécies de gramíneas forrageiras tropicais que se apresentam como opções para a formação de pastagens no Brasil (MONTEIRO et al., 1995). Para tal, o emprego de sementes de alta qualidade é fundamental para o estabelecimento rápido de plântulas no campo, propiciando um estande uniforme. A produção de sementes de alta qualidade é dificultada na maior parte das espécies forrageiras, devido ao grande número de sementes inférteis, forma de colheita das sementes, além do alto teor de impurezas que agregam nos processos da colheita e processamento de baixa tecnologia (BONOME et al., 2006).

Apesar da grande importância do gênero Brachiaria, a qualidade das sementes é variável e os investimentos são escassos (DIAS; ALVES, 2008a). Quanto ao gênero Panicum sp., a qualidade das sementes produzidas não é confiável; quase sempre é utilizado somente o teste de germinação que pode durar cerca de 28 dias, atrasando as atividades no campo (DIAS; ALVES, 2008b). Seria necessário fazer uso de tecnologias que permitem avaliar com rapidez os lotes.

O teste de condutividade elétrica é um dos métodos mais rápidos e eficientes utilizados para avaliação da qualidade de sementes. Torna-se possível analisar uma amostra de cada vez, fornecendo como resultado a média de condutividade contida na solução em que as sementes estão embebidas ou a condutividade de cada semente em particular, cujo teste é o de condutividade individual. $\mathrm{O}$ princípio desse teste é medir a quantidade de eletrólitos que as sementes liberam na água em que estão embebidas. A quantidade de eletrólito liberada na água é proporcional ao grau de desorganização da membrana plasmática e da permeabilidade nas sementes (VIEIRA; KRZYZANOWSKI, 1999).

$\mathrm{O}$ teste de condutividade elétrica proporciona resultados rápidos e confiáveis sobre a qualidade fisiológica de lotes ou de sementes individuais. O emprego da metodologia adequada facilita a tomada de decisões frente a lotes deficientes, diminuindo os riscos e prejuízos antes da instalação da cultura em campo (DIAS; MARCOS FILHO, 1996).

Dependendo da espécie em estudo, quantidade de sementes, período de embebição, volume da solução de embebição e interações, pode-se recomendar diferentes metodologias para o teste de condutividade elétrica, com indicação de 100 sementes para $100 \mathrm{~mL}$ por 24 horas à $25^{\circ} \mathrm{C}$ para sementes de milheto (GASPAR; NAKAGAWA, 2002). Pesquisa realizada com Macrotyloma axillare constatou que nas sementes dessa forrageira o período de embebição deva ser acima de 48 horas a fim de se obter a diferenciação entre lotes de sementes escarificadas (PAIVA et al., 2008).

Dias e Marcos Filho (1996) concluíram que para avaliação do vigor de sementes de soja é necessário que se realize o teste de condutividade elétrica de sementes em massa, possibilitando a redução do período de condicionamento para períodos de 4 a 8 horas evidenciando diferenças acentuadas entre os lotes. É importante que este teste seja conduzido com sementes puras.

O teste de condutividade elétrica tem alcançado resultados satisfatórios na separação de lotes de sementes com diferentes níveis de vigor para algumas culturas como a pimenta (VIDIGAL et al., 2008), ervilha (MACHADO et al., 2011), cubiu (PEREIRA; MARTINS FILHO, 2012), aveia-preta (MENEZES et al. 2007, NOGUEIRA et al., 2013) e azevém (LOPES; FRANKE, 2010).

Torna-se clara a necessidade de ajustar a metodologia para as diferentes espécies a fim de obter informações confiáveis visto que diversos fatores podem interferir nos resultados. O objetivo do estudo realizado foi adequar o teste de condutividade elétrica para melhor avaliação do vigor das sementes de Brachiaria brizantha cv. MG-5 e de Panicum maximum cv. Tanzânia.

\section{Material e Métodos}

O trabalho foi desenvolvido na Universidade Estadual de Mato Grosso do Sul no município de Cassilândia - MS. Para a pesquisa foram utilizadas sementes de Brachiaria brizantha cv. MG-5 e de Panicum maximum cv. Tanzânia, com três níveis de deterioração.

O delineamento experimental utilizado foi $\mathrm{o}$ inteiramente casualizado em esquema fatorial $3 \times 3$, constituído por níveis de deterioração das sementes (ausência, 48 e 96 horas) e períodos de embebição (8, 16 e 24 horas) à $25^{\circ} \mathrm{C}$, com 6 repetições. Avaliou-se a condutividade elétrica utilizando-se o delineamento experimental anterior, em três volumes de água da solução de embebição $(25,50$ e $75 \mathrm{~mL})$ e três quantidades de sementes (25, 50 e 75 sementes).

Os diferentes níveis de deterioração das sementes foram obtidos através de envelhecimento artificial (ausência, 48h e 96h). O envelhecimento artificial foi realizado pelo método do gerbox adaptado de Marcos Filho (1999), onde as sementes permaneceram em câmara regulada à temperatura de $41{ }^{\circ} \mathrm{C}$ durante os períodos pré-estabelecidos.

Antes da avaliação da condutividade elétrica as sementes de $B$. brizantha cv. MG-5 e P. maximum cv. Tanzânia nos três níveis de deterioração (ausência, 48 e 96 horas) foram avaliadas com um teste de emergência de plântulas, onde verificaram 86\%; 70\%, 58\% de emergência para a cultivar MG-5 para os três níveis de deterioração e $86 \% ; 70 \%$ e $58 \%$ de emergência para a cultivar Tanzânia nos três níveis de deterioração. O grau 
de umidade das sementes foi de $13,7 \%$ antes da instalação do teste da condutividade elétrica.

Para avaliação da condutividade elétrica da solução de embebição de sementes foi utilizado o teste de "condutividade de massa" ou sistema de copo. Realizada por meio de seis sub-amostras (repetições), colocadas para embeber em copos plásticos de $200 \mathrm{~mL}$, os mesmos foram mantidos à $25^{\circ} \mathrm{C}$. As leituras da condutividade elétrica foram realizadas com o condutivímetro Marconi CA 150, e os valores obtidos, foram expressos em $\mathrm{uS} \mathrm{cm} \mathrm{cm}^{-1} \mathrm{~g}^{-1}$.

Todos os dados, foram avaliados por meio da análise de variância pelo teste $\mathrm{F}$. Quando o valor de $\mathrm{F}$ foi significativo ao nível de $5 \%$ de probabilidade, aplicouse o teste de Tukey ao nível de $5 \%$ de probabilidade para comparação das médias. Considerando-se que período de embebição é uma variável quantitativa, teste de comparação de médias não é o procedimento mais adequado é a regressão polinomial pois testes de comparação de médias pode conduzir a conclusões equivocadas.

\section{Resultados e Discussão}

Para a espécie $B$. brizantha cv. MG-5 verificou-se que o uso de 25 sementes (Tabela 1), independente da quantidade de solução de embebição utilizada no teste de condutividade elétrica, no período de 8 horas, já consegue diferenciar os níveis de deterioração das sementes. O período de 16 horas não foi eficiente na diferenciação dos níveis de deterioração das sementes; já no período de 24 horas o uso de 25 ou $50 \mathrm{~mL}$ de solução de embebição, proporcionou diferenciação dos níveis de vigor. Utilizando 25 sementes (escarificadas) da espécie Macrotyloma axillare para 25 ou $50 \mathrm{~mL}$ de solução é necessário que fique embebida durante 48 horas; somente a partir desse período é possível diferenciar os lotes (PAIVA et al., 2008).

Utilizando 50 sementes de B. brizantha cv. MG-5 (Tabela 2), no período de 16 horas com 50 ou $75 \mathrm{~mL}$ de solução de embebição, evidenciou-se diferenciação dos níveis de deterioração. Em um período de 8 horas somente o uso de $25 \mathrm{~mL}$ de solução de embebição propiciou a diferenciação dos níveis de deterioração.

Vazquez et al. (2011), ao estudarem os períodos de 6,18 e 24 horas e duas temperaturas de embebição 20 ${ }^{\circ} \mathrm{C}$ e $25^{\circ} \mathrm{C}$, usando-se 50 sementes em $75 \mathrm{~mL}$ de água deionizada, verificaram que o período de $24 \mathrm{~h}$ à $25^{\circ} \mathrm{C}$ permite uma classificação dos lotes de sementes de sorgo em níveis de vigor diferentes. Ribeiro et al. (2009) observaram a possibilidade do uso do período de 24 horas com $75 \mathrm{~mL}$ de água, usando-se 50 sementes, para diferenciação do vigor de sementes de milhopipoca.

Independente da quantidade de solução de embebição utilizada no teste de condutividade elétrica B. brizantha cv. MG-5 (Tabela 3), no período de 8 horas (75 sementes) pôde-se diferenciar os níveis de vigor das sementes, resultados semelhantes obtidos com 25 sementes (Tabela 1).

Tabela 1. Condutividade elétrica $\left(\mu \mathrm{S} \mathrm{cm} \mathrm{c}^{-1} \mathrm{~g}^{-1}\right)$, de sementes de B. brizantha cv. MG-5 em função dos níveis de deterioração e períodos de embebição á $25^{\circ} \mathrm{C}$. Cassilândia (MS), 2011.

\begin{tabular}{|c|c|c|c|}
\hline \multicolumn{4}{|c|}{ Períodos de embebição (horas) } \\
\hline \multicolumn{4}{|c|}{25 sementes imersas em $25 \mathrm{~mL}$ de água } \\
\hline Níveis de deterioração & $8 \mathrm{~h}$ & $16 \mathrm{~h}$ & $24 \mathrm{~h}$ \\
\hline Ausência & ${ }^{1} 92,08 \mathrm{cB}$ & $107,20 \mathrm{cA}$ & $103,29 \mathrm{aA}$ \\
\hline $48 \mathrm{~h}$ & $146,81 \mathrm{bC}$ & $202,12 \mathrm{aA}$ & $184,96 \mathrm{bB}$ \\
\hline $96 \mathrm{~h}$ & $230,92 \mathrm{aA}$ & $188,49 \mathrm{bB}$ & $223,87 \mathrm{cA}$ \\
\hline \multicolumn{4}{|l|}{$\mathrm{CV}(\%)$} \\
\hline \multicolumn{4}{|c|}{25 sementes imersas em $50 \mathrm{~mL}$ de água } \\
\hline Níveis de deterioração & $8 \mathrm{~h}$ & $16 \mathrm{~h}$ & $24 \mathrm{~h}$ \\
\hline Ausência & $62,14 \mathrm{aB}$ & $72,97 \mathrm{bA}$ & $58,79 \mathrm{cB}$ \\
\hline $48 \mathrm{~h}$ & $73,23 \mathrm{bC}$ & $102,97 \mathrm{aA}$ & $88,42 \mathrm{bB}$ \\
\hline $96 \mathrm{~h}$ & $93,21 \mathrm{cB}$ & $107,78 \mathrm{aA}$ & $110,99 \mathrm{aA}$ \\
\hline $\mathrm{CV}(\%)$ & & 4,81 & \\
\hline \multicolumn{4}{|c|}{25 sementes imersas em $75 \mathrm{~mL}$ de água } \\
\hline Níveis de deterioração & $8 \mathrm{~h}$ & $16 \mathrm{~h}$ & $24 \mathrm{~h}$ \\
\hline Ausência & $35,61 \mathrm{cB}$ & $38,14 \mathrm{bAB}$ & $41,25 \mathrm{bA}$ \\
\hline $48 \mathrm{~h}$ & $57,65 \mathrm{bB}$ & $69,31 \mathrm{aA}$ & $68,90 \mathrm{aA}$ \\
\hline $96 \mathrm{~h}$ & $74,70 \mathrm{aA}$ & $72,18 \mathrm{aA}$ & $64,33 \mathrm{aB}$ \\
\hline $\mathrm{CV}(\%)$ & & 5,99 & \\
\hline
\end{tabular}

${ }^{\mathrm{T}}$ Médias seguidas de letras diferentes minúsculas nas colunas no fator níveis de deterioração e maiúsculas nas linhas no fator períodos de embebição, diferem estatisticamente entre si pelo teste de Tukey a 5\%. 
Tabela 2. Condutividade elétrica $\left(\mu \mathrm{S} \mathrm{m}^{-1} \mathrm{~g}^{-1}\right)$, de sementes de Brachiaria brizantha cv. MG-5 em função dos níveis de deterioração e períodos de embebição á $25^{\circ} \mathrm{C}$. Cassilândia (MS), 2011

\begin{tabular}{|c|c|c|c|}
\hline \multicolumn{4}{|c|}{ Períodos de embebição (horas) } \\
\hline \multicolumn{4}{|c|}{50 sementes imersas em $25 \mathrm{~mL}$ de água } \\
\hline Níveis de deterioração & $8 \mathrm{~h}$ & $16 \mathrm{~h}$ & $24 \mathrm{~h}$ \\
\hline Ausência & ${ }^{1} 104,26$ c B & $135,64 \mathrm{~b} \mathrm{~A}$ & 108,94 c B \\
\hline $48 \mathrm{~h}$ & $145,77 \mathrm{~b} \mathrm{~B}$ & 185,99 a A & $162,54 \mathrm{~b} \mathrm{~B}$ \\
\hline $96 \mathrm{~h}$ & 191,72 a A & 172,60 a B & 207,51 a A \\
\hline $\mathrm{CV}(\%)$ & & 8,59 & \\
\hline \multicolumn{4}{|c|}{50 sementes imersas em $50 \mathrm{~mL}$ de água } \\
\hline Níveis de deterioração & $8 \mathrm{~h}$ & $16 \mathrm{~h}$ & $24 \mathrm{~h}$ \\
\hline Ausência & $45,38 \mathrm{~b} \mathrm{~A}$ & $46,89 \mathrm{c} \mathrm{A}$ & $47,69 \mathrm{c} \mathrm{A}$ \\
\hline $48 \mathrm{~h}$ & 91,76 a B & 82,93 b C & 98,23 a $\mathrm{A}$ \\
\hline $96 \mathrm{~h}$ & 95,89 a A & 91,76 a $\mathrm{AB}$ & 88,49 b B \\
\hline $\mathrm{CV}(\%)$ & & 5,27 & \\
\hline \multicolumn{4}{|c|}{50 sementes imersas em $75 \mathrm{~mL}$ de água } \\
\hline Níveis de deterioração & $8 \mathrm{~h}$ & $16 \mathrm{~h}$ & $24 \mathrm{~h}$ \\
\hline Ausência & $26,84 \mathrm{~b} \mathrm{~B}$ & $32,69 \mathrm{c} \mathrm{A}$ & 31,48 c A \\
\hline $48 \mathrm{~h}$ & 54,83 a B & $54,54 \mathrm{~b}$ B & 67,27 a $\mathrm{A}$ \\
\hline $96 \mathrm{~h}$ & 56,05 a B & 65,42 a A & $51,44 \mathrm{~b} \mathrm{C}$ \\
\hline $\mathrm{CV}(\%)$ & & 6,58 & \\
\hline
\end{tabular}

${ }^{\mathrm{I}}$ Médias seguidas de letras diferentes minúsculas nas colunas no fator níveis de deterioração e maiúsculas nas linhas no fator períodos de embebição, diferem estatisticamente entre si pelo teste de Tukey a 5\%.

Tabela 3. Condutividade elétrica $\left(\mu \mathrm{S} \mathrm{cm} \mathrm{cm}^{-1} \mathrm{~g}^{-1}\right)$, de sementes de Brachiaria brizantha cv. MG-5 em função dos níveis de deterioração e períodos de embebição á $25^{\circ} \mathrm{C}$. Cassilândia (MS), 2011

\begin{tabular}{|c|c|c|c|}
\hline \multicolumn{4}{|c|}{ Períodos de embebição (horas) } \\
\hline \multicolumn{4}{|c|}{75 sementes imersas em $25 \mathrm{~mL}$ de água } \\
\hline Níveis de deterioração & $8 \mathrm{~h}$ & $16 \mathrm{~h}$ & $24 \mathrm{~h}$ \\
\hline Ausência & ${ }^{1} 109,01$ c B & $119,88 \mathrm{~b} \mathrm{~A}$ & $124,20 \mathrm{c} \mathrm{A}$ \\
\hline $48 \mathrm{~h}$ & $175,11 \mathrm{~b} \mathrm{~B}$ & 182,18 a $\mathrm{AB}$ & $188,08 \mathrm{~b} \mathrm{~A}$ \\
\hline $96 \mathrm{~h}$ & 202,60 a A & 188,28 a B & 203,76 a $\mathrm{A}$ \\
\hline $\mathrm{CV}(\%)$ & & 4,02 & \\
\hline \multicolumn{4}{|c|}{75 sementes imersas em $50 \mathrm{~mL}$ de água } \\
\hline Níveis de deterioração & $8 \mathrm{~h}$ & $16 \mathrm{~h}$ & $24 \mathrm{~h}$ \\
\hline Ausência & $54,00 \mathrm{c} \mathrm{A}$ & 47,74 b B & 57,59 b A \\
\hline $48 \mathrm{~h}$ & 87,78 b A & 90,98 a A & 92,97 a A \\
\hline $96 \mathrm{~h}$ & 94,09 a A & 93,32 a A & 94,15 a A \\
\hline $\mathrm{CV}(\%)$ & & 4,94 & \\
\hline \multicolumn{4}{|c|}{75 sementes imersas em $75 \mathrm{~mL}$ de água } \\
\hline Níveis de deterioração & $8 \mathrm{~h}$ & $16 \mathrm{~h}$ & $24 \mathrm{~h}$ \\
\hline Ausência & 32,14 c B & $33,87 \mathrm{~b} \mathrm{AB}$ & $36,65 \mathrm{~b} \mathrm{~A}$ \\
\hline $48 \mathrm{~h}$ & 49,49 b B & 61,61 a $\mathrm{A}$ & 61,93 a A \\
\hline $96 \mathrm{~h}$ & 65,36 a $\mathrm{A}$ & 61,35 a B & 65,65 a A \\
\hline $\mathrm{CV}(\%)$ & & 5,30 & \\
\hline
\end{tabular}

${ }^{\mathrm{T}}$ Médias seguidas de letras diferentes minúsculas nas colunas no fator níveis de deterioração e maiúsculas nas linhas no fator períodos de embebição, diferem estatisticamente entre si pelo teste de Tukey a 5\%.

Em período de 16 horas, independente da quantidade de solução de embebição, não proporcionou adequada diferenciação dos níveis de deterioração das sementes. Em período de 24 horas de embebição somente o uso de $25 \mathrm{~mL}$ de solução proporcionou a diferenciação dos níveis de vigor das sementes de maneira satisfatória.

Em relação ao período de embebição pôde-se observar maior condutividade elétrica com 24 horas de embebição em relação a 8 horas nos lotes com ausência de deterioração e por $48 \mathrm{~h}$ embebidos com 25 e $75 \mathrm{~mL}$ (Tabelas 2 e 3 ) evidenciando o fato de que quanto maior o tempo em que as sementes ficam embebidas maior a quantidade de lixiviados.

Para espécie $P$. maximum cv. Tanzânia verificou-se que a utilização de 25 sementes (Tabela 4 ), embebidas a 25 e $50 \mathrm{~mL}$ durante 16 e 24 horas proporcionou diferenciação entre os níveis de deterioração das sementes. No período de 8 horas não foi eficiente, pois somente com $50 \mathrm{~mL}$ de solução foi possível diferenciar os lotes. Para se identificar a diferença mais acentuada de lotes de sementes de soja curtos períodos entre 4 a 8 horas são suficientes (DIAS; MARCOS FILHO, 1996). 
A utilização de 50 sementes $P$. maximum (Tabela 5), durante os períodos de 8,16 e 24 horas foi eficiente para diferenciação dos lotes somente para as quantidades de 25 e $75 \mathrm{~mL}$ de solução de embebição, em soluções de $50 \mathrm{~mL}$ períodos de 16 e 24 horas.

Martins et al. (2002) constataram que para sementes pequenas como a de couve, o período de embebição pode ser bem menor do que o período utilizado para cereais ou leguminosas; 8 horas de embebição já é o suficiente para que se realize a leitura.

Ao utilizar 75 sementes $P$. maximum (Tabela 6) as possibilidades de identificação dos lotes são menores; somente com 50 e $75 \mathrm{~mL}$ de solução em um período de 24 horas foi possível realizar essa diferenciação.

Com $50 \mathrm{~mL}$ de solução foi possível fazer a diferenciação dos lotes somente no período de 8 horas, e no período de 16 horas não possibilitou diferenciação para nenhuma quantidade de solução. Para sementes de abobrinha, estudos concluíram que 50 sementes em 75 $\mathrm{mL}$ de solução seja o procedimento mais indicado para identificar lotes no período de 8 horas (DUTRA; VIEIRA, 2006).

Tabela 4. Condutividade elétrica $\left(\mu \mathrm{S} \mathrm{cm} \mathrm{c}^{-1}\right)$, de sementes de Panicum maximum cv. Tanzânia em função dos níveis de deterioração e períodos de embebição á $25^{\circ} \mathrm{C}$. Cassilândia (MS), 2011

\begin{tabular}{|c|c|c|c|}
\hline \multicolumn{4}{|c|}{ Períodos de embebição (horas) } \\
\hline \multicolumn{4}{|c|}{25 sementes imersas em $25 \mathrm{~mL}$ de água } \\
\hline Níveis de deterioração & $8 \mathrm{~h}$ & $16 \mathrm{~h}$ & $24 \mathrm{~h}$ \\
\hline Ausência & ${ }^{1} 125,59 \mathrm{~b} \mathrm{C}$ & 101,82 c A & $119,63 \mathrm{c} \mathrm{B}$ \\
\hline $48 \mathrm{~h}$ & $121,67 \mathrm{~b} \mathrm{C}$ & 148,48 b B & $173,92 \mathrm{~b} \mathrm{~A}$ \\
\hline $96 \mathrm{~h}$ & 356,35 a $\mathrm{A}$ & 459,10 a B & 415,66 a $\mathrm{A}$ \\
\hline $\mathrm{CV}(\%)$ & & 2,09 & \\
\hline \multicolumn{4}{|c|}{25 sementes imersas em $50 \mathrm{~mL}$ de água } \\
\hline Níveis de deterioração & $8 \mathrm{~h}$ & $16 \mathrm{~h}$ & $24 \mathrm{~h}$ \\
\hline Ausência & 47,90 с C & $75,25 \mathrm{c} \mathrm{A}$ & 63,89 с B \\
\hline $48 \mathrm{~h}$ & 59,82 b C & $92,30 \mathrm{~b} A$ & $75,33 \mathrm{~b} \mathrm{~B}$ \\
\hline $96 \mathrm{~h}$ & 160,50 a C & 313,50 a $\mathrm{A}$ & 222,66 a B \\
\hline $\mathrm{CV}(\%)$ & & 6,30 & \\
\hline \multicolumn{4}{|c|}{25 sementes imersas em $75 \mathrm{~mL}$ de água } \\
\hline Níveis de deterioração & $8 \mathrm{~h}$ & $16 \mathrm{~h}$ & $24 \mathrm{~h}$ \\
\hline Ausência & $36,57 \mathrm{~b} \mathrm{~B}$ & $18,72 \mathrm{~b} \mathrm{~A}$ & $13,70 \mathrm{~b} \mathrm{C}$ \\
\hline $48 \mathrm{~h}$ & $42,76 \mathrm{~b} A$ & 23,43 b B & 14,87 a B \\
\hline $96 \mathrm{~h}$ & 105,23 a A & 159,79 a B & 50,58 a C \\
\hline $\mathrm{CV}(\%)$ & & 8,77 & \\
\hline
\end{tabular}

${ }^{\mathrm{T}}$ Médias seguidas de letras diferentes minúsculas nas colunas no fator níveis de deterioração e maiúsculas nas linhas no fator períodos de embebição, diferem estatisticamente entre si pelo teste de Tukey a 5\%.

Tabela 5. Condutividade elétrica $\left(\mu \mathrm{S} \mathrm{cm} \mathrm{cm}^{-1}\right)$, de sementes de Panicum maximum cv. Tanzânia em função de níveis de deterioração e períodos de embebição á $25^{\circ} \mathrm{C}$. Cassilândia (MS), 2011

\begin{tabular}{|c|c|c|c|}
\hline \multicolumn{4}{|c|}{ Períodos de embebição (horas) } \\
\hline \multicolumn{4}{|c|}{50 sementes imersas em $25 \mathrm{~mL}$ de água } \\
\hline Níveis de deterioração & $8 \mathrm{~h}$ & $16 \mathrm{~h}$ & $24 \mathrm{~h}$ \\
\hline Ausência & ${ }^{1} 93,74$ с B & 95,13 с B & $105,26 \mathrm{c} \mathrm{A}$ \\
\hline $48 \mathrm{~h}$ & 120,34 b B & $131,31 \mathrm{~b} \mathrm{~B}$ & 166,49 b A \\
\hline $96 \mathrm{~h}$ & 383,05 a B & 386,30 a $\mathrm{AB}$ & 410,83 a A \\
\hline $\mathrm{CV}(\%)$ & & 3,90 & \\
\hline \multicolumn{4}{|c|}{50 sementes imersas em $50 \mathrm{~mL}$ de água } \\
\hline Níveis de deterioração & $8 \mathrm{~h}$ & $16 \mathrm{~h}$ & $24 \mathrm{~h}$ \\
\hline Ausência & 57,58 b B & 61,90 с C & 58,58 с A \\
\hline $48 \mathrm{~h}$ & $58,53 \mathrm{~b} \mathrm{C}$ & 69,99 b B & 89,26 b A \\
\hline $96 \mathrm{~h}$ & 178,49 a A & 122,01 a A & 214,00 a $\mathrm{A}$ \\
\hline $\mathrm{CV}(\%)$ & & 4,31 & \\
\hline \multicolumn{4}{|c|}{50 sementes imersas em $75 \mathrm{~mL}$ de água } \\
\hline Níveis de deterioração & $8 \mathrm{~h}$ & $16 \mathrm{~h}$ & $24 \mathrm{~h}$ \\
\hline Ausência & 25,67 c B & 30,99 c A & 22,84 c B \\
\hline $48 \mathrm{~h}$ & 36,45 b B & 42,93 b A & 28,16 b C \\
\hline $96 \mathrm{~h}$ & 108,11 a B & 158,51 a A & 109,08 a B \\
\hline $\mathrm{CV}(\%)$ & & 5,72 & \\
\hline
\end{tabular}

${ }^{\mathrm{T}}$ Médias seguidas de letras diferentes minúsculas nas colunas no fator níveis de deterioração e maiúsculas nas linhas no fator períodos de embebição, diferem estatisticamente entre si pelo teste de Tukey a 5\%. 
Tabela 6. Condutividade elétrica $\left(\mu \mathrm{S} \mathrm{cm} \mathrm{c}^{-1} \mathrm{~g}^{-1}\right)$, de sementes de Panicum maximum cv. Tanzânia em função dos níveis de deterioração e períodos de embebição á $25^{\circ} \mathrm{C}$. Cassilândia (MS), 2011

\begin{tabular}{|c|c|c|c|}
\hline \multicolumn{4}{|c|}{ Períodos de embebição (horas) } \\
\hline \multicolumn{4}{|c|}{75 sementes imersas em $25 \mathrm{~mL}$ de água } \\
\hline Níveis de deterioração & $8 \mathrm{~h}$ & $16 \mathrm{~h}$ & $24 \mathrm{~h}$ \\
\hline Ausência & ${ }^{1} 99,88$ b A & $105,71 \mathrm{~b} \mathrm{~B}$ & $102,61 \mathrm{~b} \mathrm{~A}$ \\
\hline $48 \mathrm{~h}$ & $102,32 \mathrm{~b} \mathrm{~B}$ & $110,97 \mathrm{~b} \mathrm{~A}$ & 104,74 b B \\
\hline $96 \mathrm{~h}$ & 306,53 a A & 192,85 a A & 311,05 a A \\
\hline $\mathrm{CV}(\%)$ & & 2,64 & \\
\hline \multicolumn{4}{|c|}{75 sementes imersas em $50 \mathrm{~mL}$ de água } \\
\hline Níveis de deterioração & $8 \mathrm{~h}$ & $16 \mathrm{~h}$ & $24 \mathrm{~h}$ \\
\hline Ausência & 56,75 c A & 59,52 b C & 65,33 c B \\
\hline $48 \mathrm{~h}$ & $67,54 \mathrm{~b} \mathrm{~B}$ & 60,11 b B & 79,79 b A \\
\hline $96 \mathrm{~h}$ & 199,30 a A & 125,33 a B & $140,52 \mathrm{a} \mathrm{AB}$ \\
\hline $\mathrm{CV}(\%)$ & & 4,85 & \\
\hline \multicolumn{4}{|c|}{75 sementes imersas em $75 \mathrm{~mL}$ de água } \\
\hline Níveis de deterioração & $8 \mathrm{~h}$ & $16 \mathrm{~h}$ & $24 \mathrm{~h}$ \\
\hline Ausência & 36,48 b A & $31,88 \mathrm{~b} \mathrm{C}$ & 23,43 c B \\
\hline $48 \mathrm{~h}$ & $39,55 \mathrm{~b} \mathrm{~A}$ & $32,83 \mathrm{~b} \mathrm{~B}$ & 33,70 b B \\
\hline $96 \mathrm{~h}$ & 135,46 a A & 94,19 a A & 104,74 a B \\
\hline $\mathrm{CV}(\%)$ & & 5,85 & \\
\hline
\end{tabular}

${ }^{1}$ Médias seguidas de letras diferentes minúsculas nas colunas no fator níveis de deterioração e maiúsculas nas linhas no fator períodos de embebição, diferem estatisticamente entre si pelo teste de Tukey a 5\%.

Ficou evidente que em sementes de B. brizantha e em sementes de $P$. maximum o período de 8 horas de embebição foi suficiente para que as sementes apresentassem boa reorganização das células e reparo de danos nas membranas; a partir desse período a liberação de exsudatos na solução em que foi embebida obteve pouca oscilação.

Nas condições em o experimento foi conduzido, o teste de condutividade elétrica de sementes de $B$. brizantha cv. MG-5 com 25 ou 75 sementes, no período de 8 horas, independente da quantidade de solução, revelou-se como melhor opção para avaliação do vigor de sementes. Já para sementes de $P$. maximum cv. Tanzânia a utilização de 50 sementes em $25 \mathrm{~mL}$ de solução, independente do período, obteve maior sensibilidade para se avaliar os níveis de deterioração.

\section{Conclusões}

Recomenda-se para Brachiaria brizantha cv. MG-5 o uso de 25 sementes no período de 8 horas no teste de condutividade elétrica, independentemente da quantidade de solução de embebição.

Para a espécie Panicum maximum cv. Tanzânia é adequado o uso de 50 sementes em $25 \mathrm{~mL}$ de solução independente do período de embebição

\section{Agradecimentos}

Os autores agradecem ao Programa de Iniciação Científica da Universidade Estadual de Mato Grosso do Sul pela concessão de bolsa ao primeiro autor.

\section{Referências Bibliográficas}

BONOME, L. T. S., GUIMARÃES, R. M., OLIVEIRA, J. A., ANDRADE, V. C.; CABRAL, P. S. Efeito do condicionamento osmótico em sementes de Brachiaria brizantha cv. Marandu. Revista Ciência e Agrotecnologia, Lavras-MG, v. 30, n. 3, p. 422-428, 2006 http://dx.doi.org/10.1590/S1413-70542006000300006.

DIAS, M. C. L. L.; ALVES, S. J. Avaliação da viabilidade de sementes de Brachiaria brizantha (Hochst. ex A. Rich) Stapf pelo teste de tetrazólio. Revista Brasileira de Sementes, Londrina-PR, v. 30, n. 3, p. 145-151, 2008 http://dx.doi.org/10.1590/S0101-31222008000300019.

DIAS, M. C. L. L.; ALVES, S. J. Avaliação da viabilidade de sementes de Panicum maximum Jacq pelo teste de tetrazólio. Revista Brasileira de Sementes, Londrina-PR, v. 30, n. 3, p. 152-158, 2008. http://dx.doi.org/10.1590/S010131222008000300020

DIAS, D. C. F. S.; MARCOS FILHO, J. Testes de condutividade elétrica para avaliação do vigor de sementes de soja (Glycine $\max$ (L.) Merrill). Revista Scientia Agricola, Piracicaba-SP, v. 53, n. 1, p. 31-42, 1996. http://dx.doi.org/10.1590/S0103-90161996000100005.

DUTRA, A. S.; VIEIRA, R. D. Teste de condutividade elétrica para a avaliação do vigor de sementes de abobrinha. Revista Brasileira de Sementes, Londrina-PR, v. 28, n. 2, p.117-122, 2006. http://dx.doi.org/10.1590/S010131222006000200015

GASPAR, C. M.; NAKAGAWA, J. Teste de condutividade elétrica em função do número de sementes e da quantidade de água para sementes de milheto. Revista Brasileira de Sementes, Londrina-PR, v. 24, n. 2, p.70-76, 2002. http://dx.doi.org/10.1590/S0101-31222002000100012. 
LOPES, R. R.; FRANKE, L. B. Teste de condutividade elétrica para avaliação da qualidade fisiológica de sementes de azevém (Lolium multiflorum L.). Revista Brasileira de Sementes, Londrina-PR, v. 32, n. 1, p. 123-130, 2010.

MACHADO, C. G.; MARTINS, C. C.; SANTANA, D. G.; CRUZ, S. C. S.; OLIVEIRA, S. S. C. Adequação do teste de condutividade elétrica para sementes de Pisum sativum subsp. Arvense. Ciência Rural, Santa Maria-RS, v. 41, n. 6, p. 988995, 2011. http://dx.doi.org/10.1590/S010384782011005000062 .

MARCOS FILHO, J. Teste de envelhecimento acelerado. In: KRYZANOWSKI, F. C.; VIEIRA, R. D.; FRANÇA NETO, J. B. (Ed.). Vigor de sementes: conceitos e testes. Londrina-PR: ABRATES, cap. 3, p. 3.1-3.21, 1999.

MARTINS, C. C.; MARTINELLI-SENEME, A.; CASTRO, M. M.; NAKAGAWA, J.; CAVARIANI, C. Comparação entre métodos para avaliação do vigor de lotes de sementes de couve-brócolos (Brassica oleracea L. var. italica Plenk). Revista Brasileira de Sementes, Londrina-PR, v. 4, n. 2, p. 96-101, 2002. http://dx.doi.org/10.1590/S010131222002000100016 .

MENEZES, N. L.; GARCIA, D. C.; BAHRY, C. A.; MATTIONI, N. M. Teste de condutividade elétrica em sementes de aveia preta. Revista Brasileira de Sementes, Londrina-PR, v. 29, n. 2, p. 138-142, 2007. http://dx.doi.org/10.1590/S0101-31222007000200019.

MONTEIRO, F. A.; RAMOS, A. K. B.; CARVALHO, D. D.; ABREU, J. B. R.; DAIUB, J. A. S.; SILVA, J. E. P.; NATALE, W. Cultivo de Brachiaria brizantha Stapf. cv. Marandu em solução nutritiva com omissões de macronutrientes. Revista Scientia Agricola, Piracicaba-SP, v. 52, n. 1, p. 135-41, 1995. http://dx.doi.org/10.1590/S010390161995000100022 .

NOGUEIRA, J. L.; SILVA, B. A.; CARVALHO, T. C.; PANOBIANCO, M. Teste de condutividade elétrica para avaliação do potencial fisiológico de sementes de aveia preta. Revista Ceres, Viçosa-MG, v. 60, n. 6, p. 896-901, 2013. http://dx.doi.org/10.1590/S0034-737X2013000600019.
PAIVA, A. S.; RODRIGUES, T. J. D.; CANCIAN, A. J.; LOPES, M. M.; FERNANDES, A. C. Qualidade física e fisiológica de sementes da leguminosa forrageira Macrotyloma axillare cv. Java. Revista Brasileira de Sementes, Londrina-PR, v. 30, n. 2, p. 130-136, 2008. http://dx.doi.org/10.1590/S0101-31222008000200016.

PEREIRA, M. D.; MARTINS FILHO, S. Adequação da metodologia do teste de condutividade elétrica para sementes de cubiu (Solanum sessiliflorum Dunal). Revista Agrarian, Dourados-MS, v. 5, n. 16, p. 93-98, 2012.

RIBEIRO, D. M.; BRAGANÇA, S.M.; GONELI, A.L.S; DIAS, D.C.F.S.; ALVARENGA, E.M. Teste de condutividade elétrica para avaliar o vigor de sementes em milho-pipoca (Zea mays L.). Revista Ceres, Viçosa-MG, v. 56, n. 6, p. 772776, 2009

VAZQUEZ, G. H.; BERTOLIN, D. C.; SPEGIORIN, C. N. Teste de envelhecimento acelerado e de condutividade elétrica para avaliar a qualidade fisiológica de sementes de sorgo (Sorghum bicolor (L.) Moench). Revista Brasileira de Biociências, Porto Alegre-RS, v. 9, n. 1, p. 18-24, 2011

VIDIGAL, D. S.; LIMA, J. S., BHERING, M. C.; DIAS, D. C. F. S. Teste de condutividade elétrica para semente de pimenta. Revista Brasileira de Sementes, Londrina-PR, v. 30, n. 1, p. 168-174, 2008. http://dx.doi.org/10.1590/S010131222008000100021 .

VIERA, R. D.; KRZYZANOWSKI, F. C. Teste de condutividade elétrica. In: KRYZANOWSKI, F. C.; VIEIRA, R. D.; FRANÇA NETO, J. B. (Ed.). Vigor de sementes: conceitos e testes. Londrina: ABRATES, cap.4, p.4.1-4.26, 1999. 